FORMATION Formation emploi

Revue française de sciences sociales

116 | octobre-décembre 2011

Pêle-mêle

\title{
La coordination européenne des politiques de l'emploi : un essoufflement depuis 2005 ?
}

Has European coordination of employment policies

been running out of steam since 2005?

Europäische Koordinierung der Beschäftigungspolitiken :seit 2005 erlahmt?

La coordinación europea de las políticas de empleo : ¿agotamiento desde 2005 ?

\section{Christine Erhel}

\section{(Q) OpenEdition}

Journals

Édition électronique

URL : http://journals.openedition.org/formationemploi/3444

DOI : 10.4000/formationemploi.3444

ISSN : 2107-0946

Éditeur

La Documentation française

Édition imprimée

Date de publication : 30 décembre 2011

Pagination : 5-18

ISSN : 0759-6340

Référence électronique

Christine Erhel, «La coordination européenne des politiques de l'emploi : un essoufflement depuis 2005 ? », Formation emploi [En ligne], 116 | octobre-décembre 2011, mis en ligne le 09 janvier 2014, consulté le 30 octobre 2020. URL : http://journals.openedition.org/formationemploi/3444 ; DOI https://doi.org/10.4000/formationemploi.3444 


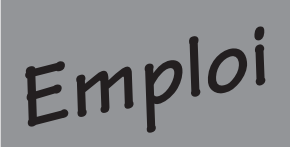

\title{
La coordination européenne des politiques de l'emploi : un essoufflement depuis 2005 ?
}

Christine Erhel

\begin{abstract}
En France, la réforme de la Stratégie Européenne pour l'Emploi ne semble pas avoir encore atteint ses ambitions en matière de participation des acteurs et de diffusion des idées européennes vers un plus large public.
\end{abstract}

Depuis 1997, et la création de la Stratégie Européenne pour l'Emploi (SEE), l'Union européenne intervient dans le domaine de l'emploi, par le biais d'une méthode de gouvernance « souple» (dite « Méthode Ouverte de Coordination » ou MOC). Cette méthode permet de pallier l'absence de compétence communautaire directe dans le domaine des politiques de l'emploi. En effet, ces politiques relèvent de la compétence des États membres en vertu du principe de subsidiarité. Cependant, selon les termes du Traité de Maastricht, l'emploi constitue une « question d'intérêt commun » et à ce titre l'Union a vocation à intervenir en appui des politiques nationales. Ce principe a été réaffirmé en 2000 et en 2005, dans le cadre de la Stratégie de Lisbonne. Celle-ci a conduit à une intégration de ces problématiques d'emploi aux questions d'éducation et de recherche, mais également aux politiques macroéconomiques (bien que celles-ci soient soumises à
* Christine Erhel est maître de conférences en économie, université Paris 1 , et membre de l'axe "Institutions » du Centre d'économie de la Sorbonne, détachée au Centre d'études de l'emploi. Thèmes de recherche : comparaisons européennes des marchés du travail et des politiques de l'emploi, Stratégie Européenne pour l'Emploi, qualité de l'emploi. Elle a publié récemment : Anxo D., Erhel C. (2008), "Irreversibility of time, reversibility of choices? The Life-Course Foundations of the Transitional Labour Marketsapproach", Revue Française de Socio- Economie, n 1. Davoine L., Erhel C. (2008), "La qualité de l'emploi en Europe : une approche comparative et dynamique ", Economie et Statistique, août. Erhel C. (201 1), "Les politiques de l'emploi en Europe : le modèle de l'activation et de la flexicurité face à la crise », Economies et Sociétés, Série SocioEconomie du Travail, n 8/201 1, pp. 1359-1388. 
des règles différentes, contraignantes pour les États membres). La stratégie Europe 2020, adoptée à l'automne $2010^{1}$, maintient également les outils de la SEE et de la MOC, dans un cadre élargi (incluant politiques d'éducation, d'environnement, de lutte contre la pauvreté, et politiques macro-économiques).

Cette intervention constitue un objet d'étude intéressant, d'une part dans l'optique de l'analyse des réformes du marché du travail et des interactions entre échelon européen et dynamiques nationales ; d'autre part, du point de vue de la gouvernance européenne, dont la MOC et la SEE constituent des formes innovantes. Certains auteurs y voient principalement un discours sans conséquence empirique ; cependant, d'autres travaux de science politique (en particulier Zeitlin, 2005) ou encore de droit (Trubek et Trubek, 2005), reposant sur des approches cognitives des politiques publiques et de la coordination, font l'hypothèse d'effets d'apprentissage et de diffusion des idées susceptibles de conduire progressivement à une forme d'harmonisation.

Toutefois, l'analyse empirique de cette coordination européenne des politiques de l'emploi pose des problèmes méthodologiques très importants. En effet, il est impossible de mettre en œuvre des analyses d'impact en isolant l'effet de la coordination européenne des autres facteurs influençant les réformes nationales. La littérature sur ce sujet propose en général des analyses qualitatives. Il s'agit alors d'identifier des effets au niveau des acteurs des politiques de l'emploi et de leur coordination. Il existe de nombreux travaux sur la première période de ce processus, avant la réforme intervenue en 2005, mais les analyses empiriques menées sur la seconde moitié des années 2000 sont rares $^{2}$. Notre objectif sera ici, à partir d'une analyse de la place des questions d'emploi dans la « relance» de la Stratégie de Lisbonne, et de ses conséquences dans la mise en œuvre de ces outils en France, d'une part, de discuter les effets de cette réforme ; d'autre

\footnotetext{
${ }^{1}$ Voir décision du Conseil du 21 octobre 2010 relative aux lignes directrices pour les politiques de l'emploi des États membres (2010/707/UE).

${ }^{2}$ Les analyses sont essentiellement conduites dans le cadre de rapports officiels (Cohen-Tanugi, 2008, pour la présidence française de l'Union européenne). De plus, la plupart des travaux existants depuis 2005 ne portent pas spécifiquement sur les questions d'emploi, mais plus largement sur la "stratégie de Lisbonne » (Begg, 2006 ; Pisani-Ferry et Sapir, 2006 ; CAS, 2008).
}

part, des hypothèses d'harmonisation cognitive via la coordination européenne. Dans une première partie, nous rappellerons les spécificités de la coordination européenne en matière d'emploi et ses évolutions dans le courant de la dernière décennie ; nous spécifierons ensuite les principales hypothèses théoriques et méthodologiques nécessaires pour les analyser. La deuxième partie propose une analyse de leurs effets à partir du cas français.

\section{LA GOUVERNANCE EUROPÉENNE DES POLITIQUES DE L'EMPLOI : HISTOIRE ET OUTILS D'ANALYSE}

La Stratégie Européenne pour l'Emploi constitue un outil récent dans le champ des politiques européennes. Elle a néanmoins connu d'importantes évolutions depuis son origine, et fait l'objet d'une abondante littérature académique sur ses effets potentiels, notamment en sciences politiques. Après avoir analysé les changements institutionnels et politiques récents, nous proposons un bref bilan des principales hypothèses existant dans cette littérature, et de leurs conséquences méthodologiques.

\section{La Stratégie Européenne pour I'Emploi : une rupture en 2005 ?}

La coordination européenne des politiques de l'emploi, dans le cadre de la Stratégie Européenne pour l'Emploi (SEE), a connu deux phases principales depuis sa création, en 1997, au Conseil de Luxembourg du 15 décembre 1997 : entre 1997 et 2004, elle fait l'objet de développements successifs, non sans critiques, avant d'être réformée en 2005 dans le cadre plus large de la relance de la Stratégie de Lisbonne.

\section{Le développement d'outils de coordination inno- vants entre 1997 et 2004}

Compte tenu de l'absence de compétence directe de l'Union dans le domaine des politiques de l'emploi'

\footnotetext{
${ }^{3}$ Depuis le Traité d'Amsterdam en 1997, la promotion de l'emploi est devenue une «question d'intérêt commun » (article 2 du Traité instituant la Communauté Européenne). La compétence de l'Union
} 
la coordination mise en place en 1997 repose sur la fixation d'objectifs communs, mais non contraignants (les lignes directrices) et sur une méthode de gouvernance innovante, dite Méthode Ouverte de Coordination (MOC). Entre 1997 et 2004, les lignes directrices pour l'emploi sont définies séparément des Grandes Orientations de Politique Economique. La MOC repose d'une part, sur un examen réciproque des plans nationaux d'action pour l'emploi (PNAE) au sein du Comité de l'Emploi ; d'autre part, sur des séances de « revue par les pairs » (avec participation d'experts de différents États membres) portant sur une politique ou une catégorie de mesures. Ce mécanisme de coordination, innovant dans sa nature, promeut une harmonisation cognitive, fondée sur la détermination d'objectifs communs et l'échange d'informations, et non sur des règles contraignantes ou des procédures incitatives.

Cette approche est confirmée et développée en plusieurs étapes au tournant des années 2000. En 2000, le Conseil européen de Lisbonne fait de la Stratégie Européenne pour l'Emploi une des composantes de la « Stratégie de Lisbonne ». Elle vise à faire de l’Union européenne "l'économie de la connaissance la plus compétitive et la plus dynamique au monde, capable d'une croissance économique durable accompagnée d'une amélioration quantitative et qualitative de l'emploi et d'une plus grande cohésion sociale ». Par ailleurs, le principe de la coordination par des objectifs communs et l'échange d'expériences entre pays, qui caractérise la MOC, est étendu à des domaines reliés aux questions d'emploi, tels que 1' "inclusion sociale » et la lutte contre la pauvreté (sommet de Nice, 2000), les retraites (sommet de Laeken, 2001), ou encore l'éducation (processus de Bologne en 1999). Cette période constitue donc une phase importante d'innovation institutionnelle et de refonte des outils des politiques européennes : pour Sabel et Zeitlin (2007), la MOC peut être analysée comme une «troisième voie » d'intervention, entre la régulation par le marché, selon le principe de libre concurrence, et l'harmonisation des politiques nationales.

est complémentaire de celle des États membres (qui restent seuls responsables de leurs politiques de l'emploi), et vise à l'élaboration d'une « stratégie coordonnée » pour l'emploi.

\section{Un affaiblissement à partir de 2004}

Malgré des évaluations souvent favorables sur le processus de la MOC, le bilan de la Stratégie de Lisbonne, dans son ensemble, est jugé décevant à mi-parcours. Cette appréciation s'appuie en particulier sur des analyses du marché du travail, notamment dans les deux rapports Kok : le premier, publié en 2003 (L'emploi, l'emploi, l'emploi : Créer plus d'emplois en Europe), reprend les orientations de la Stratégie Européenne pour l'Emploi. Il insiste cependant sur la flexibilité du marché du travail comme premier levier de création d'emploi. Le second, publié en 2004 (Facing the challenges), propose un bilan très critique de la Stratégie de Lisbonne. Elle souffrirait d'un programme trop chargé et d'une coordination insuffisante. Ce rapport plaide pour un recentrage sur la seule dimension économique. Dans l'ensemble, la réflexion menée dans ces deux rapports successifs, et au niveau de la Commission, propose une réorientation des priorités en matière d'emploi vers la flexibilisation du marché du travail ainsi qu'une articulation plus étroite entre les questions d'emploi et les orientations des politiques macro-économiques.

En pratique, ce processus critique aboutit à une « relance » de la Stratégie de Lisbonne en 2005, qui repose notamment sur un changement des modes de gouvernance dans le domaine de l'emploi. Les Lignes directrices pour l'emploi sont désormais intégrées aux Grandes Orientations de Politique Économique (GOPE), le suivi étant assuré par des « Plans Nationaux de Réforme » (PNR) pluriannuels (2005-2008; puis 2008-2010). Pour Jean-Claude Barbier (2010), cette relance correspond à un tournant au sein des politiques européennes ${ }^{4}$; ainsi, l'équilibre se déplace entre les acteurs favorables aux réformes sociales et ceux qui souhaitent un recentrage de l'UE sur les questions économiques, en faveur de ces derniers. Ce tournant résulte d'un ensemble de facteurs politiques : d'une part, l'intégration des nouveaux pays membres plus favorables à une intervention minimale dans le domaine de l'emploi et du social ; d'autre part, l'affaiblissement de la social-démocratie à cette période (y compris dans les pays du Nord qui avaient large-

\footnotetext{
4 Selon lui, il s'agit d'un « quasi effondrement » de la dimension sociale des politiques européennes, après l' « âge d'or » de 1986 à 2003 (Barbier, 2008).
} 
ment porté la création de la SEE, autour de personnalités comme Alan Larsson, homme politique suédois social-démocrate et directeur général à l'Emploi au sein de la Commission européenne entre 1995 et 2000).

Sur le fond, la période 2005-2010 est marquée par la réflexion sur la «flexicurité »; celle-ci étant définie comme l'articulation entre flexibilité du marché du travail et sécurisation des parcours par le biais des politiques actives de l'emploi et de la formation tout au long de la vie. Elle est issue d'un groupe d'experts constitué à l'initiative de la Commission, auteur d'un rapport (FlexicurityPathways) en 2007 (European Expert Group on Flexicurity, 2007). Cet objectif d'articuler flexibilité et sécurité se retrouve dans les Lignes directrices pour l'emploi. Il tend à en éluder les autres dimensions.

Par ailleurs, dans le domaine de l'emploi, les principales innovations de la période se situent en dehors du cadre de la SEE : en 2007, l'UE crée le Fonds européen d'ajustement à la mondialisation (FEM). Il vise à soutenir les travailleurs dans les entreprises en restructuration des régions et secteurs défavorisés par l'ouverture à l'économie mondialisée. Le FEM finance des mesures d'aide à la recherche d'un emploi, de recyclage sur mesure, de valorisation de l'entrepreneuriat et d'aide à l'emploi indépendant, ou encore des « compléments de revenus d'activités » spéciaux à caractère temporaire (allocations de recherche d'emploi, aides à la mobilité, aides à la formation...). L'initiative New Skills for New Jobs, lancée en décembre 2008, instaure une coopération étroite entre la Direction générale Education et la Direction génégale Emploi de la Commission européenne, autour de trois objectifs : l'amélioration des compétences, l'adéquation entre les compétences et les besoins du marché du travail, et le renforcement de la capacité d'évaluation et d'anticipation de l'Union européenne en matière de compétences ${ }^{5}$. Un rapport d'experts sur le sujet a été rendu en février 2010. Il formule une série de recommandations visant à renforcer les incitations à la formation (du côté des individus comme du côté des entreprises), à rapprocher le monde de l'édu-

5 Cf. "New skills for New Jobs. Anticipating and matching labour market needs", COM (2008) 868 Final. cation et celui du travail, à développer des compétences transversales (et transférables).

Par rapport à la période 1997-2004, la place de la SEE et de la coordination des politiques de l'emploi nationales semble plus limitée depuis 2005, les initiatives de la Commission et recommandations d'experts prenant une place plus importante. Les conséquences sur les politiques nationales de cette tendance sont incertaines, et devront être analysées dans le travail empirique.

\section{Des effets institutionnels et cognitifs}

Le processus de coordination dans le domaine de l'emploi repose sur l'idée qu'il est possible d'obtenir une convergence minimale à partir de procédures de coordination non contraignantes, fondées sur l'échange d'informations et la discussion entre les pays.

\section{La gouvernance européenne des politiques de} l'emploi : quels fondements méthodologiques et théoriques?

Toutefois, l'analyse de l'impact de cette coordination sur les politiques nationales ne peut être quantifiée, et pose de nombreux problèmes méthodologiques (Kröger, 2009). En premier lieu, le discours porté par la Stratégie Européenne pour l'Emploi apparaît flou, fondé sur des concepts dont la mise en œuvre peut prendre des formes très variées : c'est le cas par exemple de la notion d' " activation » des politiques de l'emploi, ou encore de la « flexicurité $»^{6}$. De plus, les sources d'influence et de changement politique sont multiples : au-delà des facteurs contextuels communs aux pays occidentaux (concurrence des pays à bas salaires, ou encore problèmes démographiques), d'autres institutions supranationales (comme l'OCDE - Organisation de coopération et de développement économiques) jouent un rôle très important dans la diffusion d'idées sur le marché du travail et la promotion des réformes. Enfin, les modes de gouvernance souples tels que la MOC fonctionnent à double sens : il existe également une influence du niveau national sur l'échelon européen, que l'on ne peut

${ }^{6}$ Voir sur ce point Barbier (2006), Barbier et al (2009), Gazier (2008). Pour un bilan récent, $c f$. le numéro spécial de Formation Emploi 113, janvier-mars 2011, "La flexicurité à l'aune de l'approche par les capacités ». 
appréhender à partir d'une vision purement « descendante » (top down) des politiques européennes. Pour toutes ces raisons, l'établissement d'une causalité et a fortiori d'un impact de la SEE apparaît impossible globalement. Néanmoins, une analyse des processus liés à la coordination européenne dans le domaine de l'emploi est possible, à partir de ses instruments, lignes directrices, indicateurs, rapports nationaux (PNAE et PNR), et de la mobilisation des acteurs autour de ces outils. C'est dans cette perspective, fondée sur une méthode procédurale et institutionnelle, que la SEE a en général été étudiée dans les travaux existants en science politique et en socio-économie.

Sur le plan théorique, les modes de gouvernance fondés sur la soft $\mathrm{law}^{7}$ sont susceptibles d'avoir plusieurs types d'effets. Dans une perspective juridique, Trubek et Trubek (2005) identifient six canaux : les effets de « dénonciation » (shaming) liés à la comparaison des performances nationales via des indicateurs communs (taux d'emploi par exemple) ; la diffusion au travers de l'imitation ou du discours ; les effets de délibération, lorsque la confrontation de points de vue entre les pays modifie leur approche d'un problème ; les effets d'apprentissage, c'est-à-dire l'adoption de dispositifs et de réformes sur la base des résultats obtenus par des expériences innovantes dans d'autres États-membres ; et enfin, la création de nouveaux réseaux autour des outils de gouvernance. Dans une perspective économique, en l'absence de sanctions et étant donné la faiblesse des incitations directes ${ }^{8}$, l'effet de la SEE ne peut s'analyser que dans le cadre d'approches soulignant l'importance des questions informationnelles dans la conduite des politiques publiques. Ainsi, les théories du fédéralisme budgétaire ${ }^{9}$ mettent également en avant la possibilité d'un apprentissage décentralisé se diffusant ensuite aux autres composantes du système fédéral (Oates, 1999). L'avantage de l'expérimentation décentralisée est d'autant plus important

\footnotetext{
7 Contrairement à la « hardlaw», dont le non-respect s'accompagne de sanctions, un texte crée du droit mou (soft law) quand il conseille et oriente, sans poser d'obligation juridiquement sanctionnée.

8 L'articulation de principe des financements du FSE avec la MOC peut constituer une incitation financière. Toutefois, la mise en œuvre du FSE semble en général déconnectée de la SEE à ce stade.

9 Un système fédéral est défini dans cette littérature comme un système où il existe plusieurs niveaux de gouvernement, chacun ayant une autorité politique, indépendamment de toute constitution formelle : il est donc différent du fédéralisme au sens politique du terme.
}

que l'incertitude sur l'impact des politiques est élevée (ce qui est le cas pour les politiques de l'emploi). Plus largement, les théories institutionnalistes (et en particulier North, 1990) intègrent le rôle des idées dans le changement institutionnel et politique ${ }^{10}$. Pour North, le changement est en général graduel, sans rupture par rapport à la trajectoire antérieure (selon le principe de la dépendance du sentier). Toutefois, les idées peuvent amener à des innovations correspondant à un changement de sentier de dépendance : une institution nouvelle conduit alors à l'adoption d'autres institutions complémentaires, comme en matière technologique lorsqu'une technologie nouvelle apparaît et se diffuse. Cette orientation rejoint certains travaux menés en science politique, insistant sur la dimension cognitive des politiques publiques et le rôle des idées à l'origine du changement (voir notamment Hall, 1989; Muller, 2000). Concernant la SEE, les travaux de Zeitlin (2005) insistent sur son potentiel d'expérimentation et d'apprentissage, conduisant au changement via la diffusion d'idées sur les politiques de l'emploi. Toutefois, d'autres auteurs (Kröger, 2009) soulignent dans l'ensemble que l'on ne peut s'en tenir à une analyse cognitive des effets des MOC, étant donné l'importance des jeux d'acteurs (partis politiques, mais aussi partenaires sociaux, etc.).

\section{L'impact sur la mise en œuvre et la définition des politiques nationales}

La littérature empirique existante ${ }^{11}$, fondée sur des études de cas nationaux, confirme la présence de plusieurs canaux par lesquels les MOC impactent les politiques nationales. Premièrement, les MOC ont contribué au changement dans le contenu des politiques nationales en diffusant des concepts et des catégories européennes (activation, prévention, formation tout au long de la vie, gendermainstreaming, inclusion sociale), et en modifiant l'agenda des politiques nationales. Deuxièmement, conformément à leur objectif, les MOC semblent avoir favorisé l'apprentissage mutuel ; même si, selon la littérature existante, il n'existe pas d'exemple d'emprunt direct d'une politique menée à l'étranger qui pourrait être attribué à

\footnotetext{
10 Voir Erhel et Palier (2005).

11 Pour le cas français, voir Barbier (2002), Erhel, Mandin, Palier (2005). Dans une perspective comparée, Pochet et Zeitlin (2005) et plus récemment Zeitlin et Heidenreich (2009).
} 
la SEE. Toutefois, les travaux qualitatifs ont montré qu'il existait des effets d'apprentissage et de diffusion des idées en matière d'intervention sur le marché du travail (Pochet, Zeitlin, 2005 ; Kröger, 2009), même si les déclinaisons nationales restent différentes en fonction des modèles nationaux : c'est le cas par exemple de la thématique de l'activation, devenue centrale dans les réformes des politiques d'emploi, depuis la fin des années 1990, ou encore de la "flexicurité » mise en avant depuis 2005. Troisièmement, la MOC a contribué à modifier la mise en œuvre des politiques de l'emploi nationales, via des effets de rationalisation des politiques existantes et de leurs justifications, et des effets de coordination. Le processus a conduit à une amélioration de la coordination au sein de l'administration, au niveau horizontal (entre les ministères), et vertical (entre le niveau national et les échelons locaux ou régionaux) : en ce sens, la MOC semble avoir constitué un outil de gestion interne à l'administration de l'emploi. Toujours dans le cadre des politiques nationales, la Stratégie Européenne pour l'Emploi propose une référence nouvelle. Elle permet aux acteurs de légitimer leurs positions, et le cas échéant de servir leurs intérêts dans le débat national, ce que l'on peut qualifier d' " effet de levier » (Erhel, Mandin, Palier, 2005). Enfin, la SEE a souvent conduit à un accroissement de l'implication des partenaires sociaux dans la définition des orientations des politiques de l'emploi.

Compte tenu de l'importance de ces effets institutionnels, on peut donc s'attendre à ce que la réforme des modes de gouvernance, en 2005, ait un impact sur la coordination dans le domaine de l'emploi, y compris à l'échelon national. L'intégration des lignes directrices modifie en effet la place relative des acteurs économiques par rapport aux institutions du travail et de l'emploi. C'est cette hypothèse que nous chercherons à tester grâce à un analyse empirique du cas français.

\section{UN AFFAIBLISSEMENT ENTRE 2005 ET 2010 ? UNE ANALYSE À PARTIR DU CAS FRANCCAIS}

Conformément aux orientations méthodologiques présentées précédemment, nous nous situons au niveau de la mise en œuvre du volet emploi de la Stratégie de Lisbonne. Cette stratégie repose sur les Plans Nationaux de Réforme (PNR), des procédures de discussion et de coordination autour du PNR, et sur des acteurs. En pratique, nous nous appuyons sur les documents produits dans le cadre de la Stratégie de Lisbonne, et sur des entretiens auprès des acteurs en charge de la mise en œuvre des lignes directrices pour l'emploi, réalisés en 2008-2009 (cf.encadré 1). L'objectif de cette analyse est d'identifier les effets de la Stratégie Européenne pour l'Emploi sur les institutions, la gouvernance des politiques de l'emploi, et les stratégies des acteurs. Nous nous concentrons sur la période 2005-2008, faisant suite à la relance du processus de Lisbonne (et précédant la crise). Ceci n'exclut pas une mise en perspective plus longue permettant de mettre en évidence les éléments de permanence et de changement dans les dynamiques institutionnelles.

Dans cette partie, l'analyse porte, dans un premier temps, sur la mise en œuvre, en France, des outils de la coordination européenne en matière d'emploi (PNR, institutions et acteurs) avant d'aborder leurs effets politiques (diffusion auprès du grand public, réformes).

\section{Des Plans Nationaux d'Action pour l'Emploi au Plan National de Réforme}

La présentation des premiers PNAE (entre 1997 et 2002) suivait la distinction entre les quatre piliers énoncés dans la $\mathrm{SEE}^{12}$. Elle énumérait en outre les lignes directrices ainsi que les mesures correspondantes dans les politiques de l'emploi françaises. Les dispositifs les plus importants étaient intégrés dans le cadre des lignes directrices, même lorsqu'elles représentent une spécificité française. La modification des lignes directrices, en 2003 et 2004, fut reprise dans la présentation du PNAE français qui suivait leur nouvelle formulation. En dépit du changement de majorité politique en 2002, l'interprétation des priorités européennes changea peu pendant cette période.

\footnotetext{
${ }^{12}$ Ces quatre piliers étaient les suivants : améliorer la capacité d'insertion professionnelle ou employabilité, développer l'esprit d'entreprise, encourager la capacité d'adaptation, renforcer la politique d'égalité des chances.
} 


\section{Encadré 1 \\ Analyser le cas français}

L'analyse du cas français a été réalisée dans le cadre plus large d'une étude comparative européenne de la mise en œuvre du volet emploi de la Stratégie de Lisbonne depuis 2005. Elle a été réalisée en 2008 pour cinq pays (France, Allemagne, Espagne, Pologne, Hongrie), à la demande du Parlement européen $\left({ }^{*}\right)$. Ses principaux résultats ont été repris et mis en perspective avec six pays ll'Allemagne, le Danemark, l'Italie, la Pologne, le Royaume-Uni, la Slovaquie) dans un rapport sur le même thème, pour la Direction générale Emploi de la Commission européenne $\left({ }^{* *}\right)$. Cette dernière étude comportait également un questionnaire adressé aux acteurs administratifs nationaux en charge de la SEE.

Elle repose sur deux types de sources:

(1) Une analyse des documents produits dans le cadre de la Stratégie de Lisbonne depuis 2005 (Plans Nationaux de Réforme français, rapports de suivi du PNR français, rapports conjoints sur l'emploi et recommandations de la Commission). Ces documents ont été comparés avec ceux de la période 1997-2004 (Plans Nationaux d'Action pour l'Emploi) afin de repérer les inflexions dans la présentation et le contenu.

(2) Des entretiens semi-directifs conduits auprès des acteurs en charge de la mise en œuvre du volet emploi de la Stratégie de Lisbonne (Centre d'analyse stratégique - CAS, Secrétariat général aux Affaires européennes - SGAE, Délégation aux Affaires européennes et internationales - DAEI, Mouvement des entreprises de France - MEDEF, Confédération française des travailleurs chrétiens - CFTC).

Des contacts avaient été pris avec d'autres organisations syndicales, qui n'ont pas souhaité participer à un entretien, renvoyant au rapport récemment publié de la Mission Cohen-Tanugi (l'Europe dans la Mondialisation, publié en 2008). Cette mission s'était en effet appuyée sur le Comité du Dialogue Social pour les questions européennes et internationales (CDSEI), dont l'ensemble des organisations syndicales et patronales sont membres.

Ces entretiens, d'une durée d'une heure à 1 heure 30, ont été conduits en face à face en décembre 2008 et janvier 2009. La grille d'entretien suivie comportait trois parties principales : description et appréciation du processus de mise en œuvre de la Stratégie de Lisbonne lavec une interrogation plus spécifique sur les évolutions observées depuis 2005), analyse des effets potentiels de la Stratégie sur les réformes observées en France/ou à l'inverse influence de la France sur les orientations européennes (avec des exemples, si possible récents), et enfin limites du fonctionnement actuel de la Stratégie de Lisbonne (en France et à l'échelon européen) et pistes de réforme à l'horizon 2010.

Les entretiens ont été complétés par des contacts par mail ou téléphone avec d'autres acteurs impliqués (CAS, Commission chargée des Affaires européennes à l'Assemblée nationale), mais qui n'étaient pas disponibles pour un entretien en face à face.

(*) Rodriguez R., Warmerdam J., and Triomphe C.-E. (dir.) (2010).

(**) Begg I., Erhel C., Mortensen J. (2010).

Les plans français incluaient des références à l'activation, aux incitations au travail (makingworkpay), au capital humain et à la formation tout au long de la vie, à l'égalité de genre et à la conciliation vie familiale vie professionnelle, ainsi que des considérations en termes de cohésion sociale et de lutte contre l'exclusion.

Le premier PNR de 2005 poursuit cette tendance, comme l'illustre son titre : "Pour une croissance sociale ». Mais contrairement aux PNAE précédents, il ne reprend pas l'ensemble des lignes directrices. Il propose une approche synthétique sur la base de quatre axes : l'emploi, la compétitivité des entreprises, les politiques industrielles et de recherche, et la politique budgétaire.

Le premier axe inclut deux thèmes principaux, «Créer les conditions du retour à l'emploi » et «Valoriser 
le travail ». Les rapports de suivi de 2006 et 2007 adoptent une présentation différente et synthétisent les politiques françaises en trois parties : croissance (politique budgétaire, retraites et réforme de la sécurité sociale, politiques sociales...), emploi (incitations au travail, capital humain et fonctionnement du marché du travail), et économie de la connaissance (recherche et développement, politique industrielle...). Malgré un contenu similaire, le titre de la première partie est modifié entre les deux rapports successifs : «Créer les conditions pour une croissance sociale» (2006) devient «Créer les conditions pour une croissance soutenable et durable » (2007). Le PNR 2008-2010 est également divisé en trois parties : "Croissance durable, innovation et développement des entreprises », «Des opportunités pour tous » et «Soutenabilité des finances publiques ». Les politiques de l'emploi, ainsi que les politiques sociales, sont regroupées dans la deuxième partie.

Ainsi, la structure des documents français a évolué vers une présentation plus générale et plus éloignée du texte des lignes directrices, depuis 2005. Il semble ainsi que l'adoption des lignes directrices intégrées ait conduit à une présentation large, plus proche du programme gouvernemental dans son ensemble que d'une formulation spécifiquement liée aux lignes directrices. La place accordée aux questions d'emploi devient beaucoup plus réduite dans ce nouvel outil de coordination. En termes de contenu affiché, on note que le PNR reste proche des grandes orientations européennes, mais avec un affaiblissement de la référence à la cohésion sociale, notamment dans les titres. Ceci constitue une première indication des effets possibles du changement d'acteurs intervenant dans la rédaction du PNR (désormais confiée au ministère de l'Économie en raison de l'intégration des lignes directrices, voir infra), et de l'affaiblissement du poids des acteurs sociaux dans le cadre des outils de coordination en matière d'emploi ${ }^{13}$.

\footnotetext{
${ }^{13}$ Même s'ils continuent d'intervenir en parallèle dans le cadre d'une autre MOC, dite « inclusion sociale », produisant un rapport «plan national d'action pour l'inclusion sociale » (le dernier porte sur la période 2008-2011).
}

\section{Un renforcement de la sphère économique au détriment de celle de l'emploi}

Sur le plan institutionnel, la période 2005-2008 a été marquée par des réformes dans la mise en œuvre de la stratégie de Lisbonne.

Au niveau administratif et ministériel, le Secrétariat général des Affaires européennes (SGAE) a remplacé le Secrétariat général du comité interministériel depuis le 18 octobre 2005. En dépit du changement de nom, le statut de cette institution et ses missions sont restés identiques : le SGAE est placé sous l'autorité $\mathrm{du}$ Premier ministre. Il assure une mission de coordination entre les institutions européennes et l'administration française, ainsi qu'au sein de l'administration française en charge des questions européennes. Depuis 2006, le SGAE est secondé par le Conseil d'analyse stratégique (qui a remplacé le Commissariat Général au Plan); ce dernier a un rôle d'expertise et participe au suivi du PNR. Toutefois, le processus de coordination autour de la rédaction du PNR s'est transformé en raison de réformes de la structure ministérielle intervenues en $2007^{14}$. En effet, les questions d'emploi ont été transférées du ministère du Travail au ministère de l'Économie en 2007, faisant l'objet d'un suivi par la DGTPE (Direction générale du Trésor et de la Politique économique). Du fait de l'existence de lignes directrices intégrées depuis 2005, le ministère de l'Économie était donc en charge de l'essentiel de la Stratégie de Lisbonne (politiques macro-économiques et emploi). C'est d'ailleurs Mme Lagarde, ministre de l'Économie, des Finances et de l'Emploi qui a été nommée coordinatrice nationale («Mme Lisbonne ») en juillet 2007. Ce transfert de compétences ne fut pas sans conséquences. Il conduit en effet d'une part à un affaiblissement du rôle du ministère du Travail (et tout particulièrement de la Délégation générale à l'Emploi et à la Formation professionnelle - DGEFP -, qui avait assuré l'essentiel de la dynamique française autour de la SEE entre 1997 et 2007) ; d'autre part à un affaiblissement du SGAE. En raison de l'intégration des questions d'emploi et des politiques macro-économiques, le PNR était en effet rédigé par Bercy.

\footnotetext{
${ }^{14}$ Depuis novembre 2010, les questions d'emploi sont de nouveau une compétence du ministre du Travail.
} 
"Les choses sont plus formelles depuis que l'emploi est à Bercy. Dans les réunions du SGAE, c'est la DGTPE qui a un rôle de leader, et plus la DGTEFP. Avant cela, c'était la DGTEFP qui s'occupait des lignes directrices emploi, et la DGTPE avait compétence sur les Grandes Orientations de Politique Economique. Maintenant, c'est la DGTPE qui prend tout en charge, même si le processus demeure en principe interministériel. ${ }^{15}$ "

Au niveau parlementaire, un certain nombre d'efforts ont été réalisés afin d'accroître la participation des parlementaires aux politiques européennes, et en particulier à la Stratégie de Lisbonne. Deux Commissions des Affaires européennes ont été créées au sein de l'Assemblée nationale et du Sénat, en remplacement des délégations existant auparavant, et les rapports de suivi du PNR y ont été présentés dès 2006. En ce sens, les institutions françaises ont suivi les recommandations de la relance de Lisbonne en 2005 sur la nécessité d'accroître la participation du Parlement et des personnels politiques en général. Toutefois, la publicité autour de ces questions européennes demeure très limitée parmi les parlementaires, ainsi qu'aux échelons décentralisés (région, département, municipalités).

La participation des partenaires sociaux avait été institutionnalisée en 1998 au travers du Comité du dialogue social sur les questions européennes et internationales (CDSEI). Depuis 2000, leur implication dans la rédaction des PNAE était réelle. Toutefois, les dernières années montrent des signes d'affaiblissement : en particulier, depuis 2006, les partenaires sociaux ne participent plus à la présentation du plan français à la Commission, et leurs commentaires ne sont plus inclus dans le PNR 2008-2010, contrairement à la pratique antérieure. Les explications avancées par les acteurs renvoient principalement à des facteurs politiques internes, l'élection du président de la République en 2007 ayant conduit à un recentrage du débat sur le programme présidentiel de réforme. Les réformes françaises (telles que la loi de modernisation du marché du travail en 2007) ont mobilisé les acteurs syndicaux et patronaux, au détriment du débat

15 Entretien, DAEI (Délégation aux Affaires européennes et internationales), 23-12-2008 sur les lignes directrices et leur mise en œuvre, qui apparaît « marginal ».

"Cette année, le MEDEF (Mouvement des entreprises de France) n'a pas participé au PNR. Ce n'est pas le lieu de décision, et le MEDEF pare au plus pressé. L'intervention des partenaires sociaux a lieu en amont, par exemple concernant l'accord de Janvier 2007 sur le droit du travail et la flexicurité. La SEE, c'est devenu marginal» ${ }^{16}$.

"Le CDSEI est une originalité française : on arrive à parler, il y a une réelle concertation. Depuis 2007, les ministres du Travail ne s'impliquent plus. Mais ce serait une erreur d'y toucher. Le problème principal c'est que les partenaires sociaux ne sont pas associés à la mise en cuvre ${ }^{17}$.

On peut par ailleurs faire l'hypothèse que cette participation plus limitée est aggravée par les réformes administratives : les partenaires sociaux entretiennent des liens historiquement forts avec le ministère du Travail, dont le rôle dans la Stratégie de Lisbonne et les politiques de l'emploi s'est affaibli au profit du ministère de l'Economie et des Finances dans la période 2007-2010 ${ }^{18}$.

Au-delà de changements de dénominations et d'une participation légèrement accrue du Parlement, la période récente semble marquée par un affaiblissement de l'effet de coordination mis en évidence dans des travaux antérieurs. La mise en place des lignes directrices intégrées a coïncidé avec un renforcement de la sphère des politiques économiques, au détriment de celle du travail ; elle a conduit de fait à un affaiblissement des préoccupations relatives à l'emploi autour des politiques européennes. Par ailleurs, l'affirmation forte d'un agenda national de réformes a également conduit à une focalisation quasi exclusive des acteurs sur le débat national, effaçant les enjeux européens.

\section{Un club d'européanistes?}

En France, une limite importante de la mise en œuvre de la SEE tient à sa très faible diffusion auprès du personnel politique (membres des cabinets, ministres,

\footnotetext{
16 Entretien, MEDEF, 15-01-2009.

17 Entretien, CFTC, 23-01-2009.

18 Voir supra.
} 
parlementaires, etc.), mais également syndical, ou encore de l'opinion publique.

"Le degré d'appropriation politique est faible. Il existe certes un "club" des 'lisbonniens'français. Les gens qui reçoivent les informations sont environ 150. La question est ensuite : quelle capacité d'impact sur le cabinet du ministre et le ministre ? ${ }^{19}$

"Ça reste réservé à un public d'initiés $»^{20}$.

Il apparaît que cette situation n'a que peu évolué, en dépit de l'ambition affichée de la relance de Lisbonne en 2005 d'accroître la diffusion auprès de l'opinion publique. La présidence française de l'Union européenne, en 2008, a contribué à améliorer la connaissance, par le personnel politique, des enjeux européens, y compris la Stratégie de Lisbonne et la coordination en matière d'emploi. Mais cette diffusion est demeurée limitée. Ce constat, repris par l'ensemble des acteurs, rejoint d'autres travaux antérieurs : le rapport Cohen Tanugi (2008) insiste également sur ce défaut d'appropriation, de même que l'étude de Pisani Ferry et Sapir (2006) sur la diffusion de la Stratégie de Lisbonne dans les pays de l'Union européenne. Parmi les explications potentielles de ce faible intérêt pour les questions européennes, on peut avancer la culture politique, encourageant le localisme, ou encore la taille du pays, qui accroît l'hétérogénéité interne et donc la difficulté de se coordonner sur des orientations communes. Par ailleurs, il est probable que l'usage souvent négatif de la référence aux politiques européennes dans le discours politique (l'Union européenne est le plus souvent citée comme une contrainte, limitant des marges de manœuvre des politiques nationales) a une influence sur la perception de l'Europe par les citoyens (Cohen Tanugi, 2008).

\section{Les effets de levier moindres?}

Dans notre analyse empirique, nous retrouvons les résultats des études antérieures à 2005 sur l'existence d'un effet de levier, c'est-à-dire d'une utilisation stratégique de la référence aux lignes directrices afin de promouvoir certaines orientations de réforme. Toutefois, cet effet semble moins fort depuis 2005 .

\footnotetext{
${ }^{19}$ Entretien, CAS, 09-01-2009.

${ }^{20}$ Entretien, DAEI, 23-12-2008.
}

La plupart des acteurs interviewés mentionnent des exemples antérieurs à la relance de Lisbonne (par exemple le PARE-Plan d'aide au retour à l'emploien 2001, la réforme des retraites de 2003, ou le Plan national d'action pour l'emploi des seniors). Depuis 2005, l'influence semble plus diffuse : les orientations européennes ne sont pas citées directement, même si certains rapports officiels (rapport Cahuc-Kramarz ou rapport Attali) insistent sur la nécessité de flexibiliser le contrat de travail en contrepartie d'une sécurisation des parcours, utilisant l'exemple du Danemark à l'appui de leurs propositions. L'accord de « modernisation du marché du travail », du 11 janvier 2008, a été présenté comme l'amorce d'une « flexicurité à la française ", même si l'essentiel des dispositions incluses conduisent plutôt à accroître la flexibilité sans réelle contrepartie. De plus, dans les faits, cette orientation de réforme a connu un succès très limité en France, l'idée du contrat unique ou plus généralement de la réduction de la protection de l'emploi rencontrant une opposition forte de la part des syndicats et des salariés ( $c f$. l'échec du Contrat Première Embauche), et un soutien relativement faible des représentants des employeurs. En revanche, des réformes très importantes (comme le Revenu de Solidarité Active) ne peuvent toutefois être directement reliées aux orientations européennes; dans ce cas précis, il semble que l'on assiste plutôt à une volonté de diffuser cet exemple de réforme française auprès des institutions européennes :

«En ce moment, au Comité de Protection Sociale, Martin Hirsch défend l'idée d'expérimentations sociales pour lutter contre la pauvreté, et l'exemple $d u$ RSA. Le Fonds d'ajustement à la mondialisation a également été une mesure portée par la France : 500 millions d'euros, ce n'est pas négligeable! ${ }^{21}$ "

Néanmoins, s'il ne constitue pas un exemple d'effet de la SEE et des MOC sur les politiques françaises, cet exemple atteste bien d'interactions renforcées (et à double sens) entre l'échelon national et l'échelon européen.

Par ailleurs, nos interviews suggèrent un effet « implicite » des orientations européennes, même en l'absence de référence à celles-ci dans le discours politique :

\footnotetext{
${ }^{21}$ Entretien SGAE (Secrétariat général aux affaires européennes), 3 décembre 2008
} 
"La référence n'est pas forcément explicite. Il y a une volonté des politiques de ne pas trop se référer à l'échelon européen ; mais ça structure. »

"Lisbonne, c'est un levier, un aiguillon. " ${ }^{22}$

La SEE et la Stratégie de Lisbonne auraient contribué à la définition d'un agenda de réformes commun à l'ensemble des pays européens :

"Les effets de la SEE et de la Stratégie de Lisbonne sur les réformes sont certains. Des réformes du service public de l'emploi ont eu lieu partout en Europe : suivi individualisé, prestations dégressives, guichet unique pour l'accueil des demandeurs d'emploi... Un consensus s'est construit au-delà des divergences politiques. Dès 2005, il y avait une sorte de perception commune des priorités en termes de réformes. $»^{23}$

Cet effet d'agenda se double d'un effet d'apprentissage de la comparaison internationale, qui apparaît de plus en plus utilisée, au-delà même des procédures prévues par les MOC :

"On fait plus de comparaisons: 'les Danois le font'. On met l'accent sur les bonnes pratiques, qui peuvent être plus efficaces que les lignes directrices en termes politiques. $^{24}$ 》

Dans l'ensemble, ces résultats suggèrent que la SEE a créé une dynamique institutionnelle et cognitive, qui n'est pas sans effet sur les réformes, mais cette dynamique ne s'est pas accélérée. Au contraire, ses effets apparaissent relativement stables au cours du temps : coordination administrative, apprentissage de la comparaison internationale par les acteurs politiques et sociaux, diffusion d'idées auprès d'un club d'intellectuels et d'administratifs, et quelques usages stratégiques afin de soutenir des réformes. Par rapport à ses ambitions, la relance de 2005 ne semble pas avoir favorisé une meilleure diffusion de la Stratégie de Lisbonne et de ses outils. Dans le domaine de l'emploi, le processus de fusion des lignes directrices avec les GOPE - Grandes orientations de politique économique - et l'inscription dans le PNR semblent avoir affaibli la coordination mise en place depuis 1997, en réduisant la place des acteurs de la sphère

22 Entretien DAEI 23-12-2008.

23 Entretien SGAE 03-12-2008.

24 Entretien, CAS, 09-01-2009. du travail (ministère du Travail, syndicats) au profit de la sphère économique. Le processus de la MOC elle-même apparaît moins dynamique au cours des dernières années, en dépit d'une adhésion maintenue des acteurs administratifs au processus.

Pour le cas français, les résultats apparaissent en phase avec les enquêtes de terrain réalisées dans d'autres pays, y compris les plus récentes (dans le cadre du rapport Rodriguez et al (2010), ou encore par exemple pour le rapport Begg et al (2010) ${ }^{25}$. Dans l'ensemble, les analyses révèlent une bonne adhésion des acteurs administratifs impliqués dans le processus aux principes de la MOC, mais une faible participation des acteurs de la société civile et des partenaires sociaux. Le caractère « élitiste » de ces outils de gouvernance européenne est relevé dans la plupart des pays, et ne semble pas avoir évolué favorablement depuis 2005. On retrouve également l'idée selon laquelle l'intégration des lignes directrices a souvent conduit à un effacement des questions d'emploi. Toutefois, l'appréciation de l'influence de la SEE est contrastée au sein des pays étudiés. Dans certains pays comme la Pologne, elle semble avoir constitué une source d'inspiration pour les réformes des politiques de l'emploi nationales; tandis que l'influence est très limitée dans des pays comme le Danemark ou le Royaume-Uni. En général, les thèmes les plus souvent cités pour cette influence sont le vieillissement actif, la conciliation vie familiale/vie professionnelle, la modernisation du service public de l'emploi (guichet unique, recours à des opérateurs privés) ou encore la flexicurité. Les outils d' « apprentissage mutuel » font l'objet d'appréciations positives, tandis que le processus des rapports (PNR) est critiqué pour sa complexité et son absence de lisibilité.

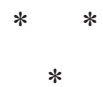

L'analyse de la gouvernance européenne des politiques de l'emploi, depuis 1997, témoigne d'un tournant en 2004-2005 : entre 2005 et 2008, la place des outils issus de la SEE apparaît plus réduite, tandis que se développent des initiatives de la Commission. Dans le cas de la France, la réforme de la Stratégie

${ }^{25} C f$. encadré 1. 
Européenne pour l'Emploi et l'intégration des lignes directrices macro-économiques et emploi ne semblent pas avoir atteint leurs ambitions en matière de participation des acteurs politiques et sociaux et de diffusion des idées européennes vers un plus large public ; même s'il a conduit de fait à une certaine simplification administrative en conférant un rôle prédominant à la sphère de l'économie. Plus largement, au-delà de cette réforme, la dynamique observable, depuis la création de la SEE, ne montre pas d'effets d'accélération; sa place dans les stratégies des acteurs apparaissant au mieux stable, voire en recul. La thèse d'une harmonisation par les idées semble avoir une portée empirique relativement limitée, et concentrée sur quelques dimensions des politiques de l'emploi (flexicurité, activation, réforme du service public de l'emploi). En référence aux travaux institutionnalistes, on serait face à une dynamique de changement institutionnel graduel et progressif, sans création d'un nouveau sentier et sans véritable changement de référentiel global en matière d'emploi.

Ces résultats, que l'on retrouve dans d'autres études nationales comparables, peuvent conduire à s'interroger sur le choix opéré dans le cadre de la Stratégie
Europe 2020. En effet, cette stratégie réduit certes le nombre de lignes directrices, mais accorde une place très importante aux questions d'éducation et de formation, au détriment de l'emploi. Celui-ci demeure pourtant une des préoccupations centrales des Européens ; selon un sondage d'opinion réalisé pour le Parlement européen (Eurobaromètre ${ }^{26}$ ) en janvier 2009, 57 \% des citoyens citent le chômage comme un thème important pour la campagne électorale européenne, devant la croissance économique, ou encore l'inflation et le pouvoir d'achat. Ce pourcentage dépasse même $80 \%$ en Irlande, en Grèce et au Portugal. Les lignes directrices étant désormais adoptées, les pistes pour améliorer la visibilité et l'efficacité de la dimension emploi des politiques européennes reposent sur l'approfondissement des outils existants (réflexion sur les indicateurs et suivi, renforcement de l'apprentissage mutuel), voire le développement, peu probable dans un avenir proche, d'outils de politiques de l'emploi harmonisés entre les pays (soutien à la mobilitée ${ }^{27}$, dispositifs d'orientation professionnelle...).

\footnotetext{
${ }^{26}$ Eurobaromètre 71, « Elections européennes 2009 ».

27 Cf. par exemple Wasmer et Waizsäcker (2007).
}

\section{Bibliographie}

Barbier J.-C. (2008), La longue marche vers l'Europe sociale, PUF, « Le lien social».

Barbier J.-C. (2010), « Stratégie de Lisbonne: les promesses sociales non tenues ", Documents de travail du CES, 2010.18

Barbier J.-C., Collomb F., Madsen P. (2009), "Flexicurity-an open method of coordination, at the national level?", Documents de travail du CES, 2009.46 .

Barbier J.-C., Samba Sylla N. (2002), La Stratégie européenne pour l'emploi : les représentations des acteurs en France, rapport du CEE.
Begg I. (2006), Lisbon relaunched, CEPS report, December.

Begg I., Erhel C., Mortensen J. (2010), Medium term employment challenges of the Lisbon strategy, № DG EMPL VT/2008/010, CEPS, Bruxelles. http:// ec.europa.eu/social/main.jsp?catId=101\&langId=en

CAS (2007), La Stratégie de Lisbonne, Une voie européenne dans la mondialisation, rapport, septembre, $46 \mathrm{p}$.

Cohen-Tanugi L. (2008), Une Stratégie Européenne pour la mondialisation, rapport de la mission "l'Europe dans la mondialisation". 
Davoine L., Erhel C., Guergoat M. (2008), "Monitoring Employment Quality in Europe: European Employment Strategy Indicators and Beyond", International Labour Review, september.

Erhel C., Mandin L., Palier B. (2005), « The Leverage Effect: the Open Method of Coordination in France », chapter 8, in Philippe Pochetet, Jonathan Zeitlin (dir).

Erhel C., Palier B. (2005), « Europe sociale et Europe de l'emploi : l'apport de Douglass North à l'explication des trajectoires nationales ", Economies et Sociétés, Série Socio-Economie du Travail, $\mathrm{n}^{\circ} 26$, 8/2005, pp. 1531-1556.

European Expert Group on Flexicurity (2007) Flexicurity Pathways: Turning hurdles into stepping stones.

Gazier B. (2008), "Flexicurité et marches transitionnels du travail", Travail et Emploi, $\mathrm{n}^{\circ} 113$, pp. 117-128.

Hall P. (1989), The Political Power of Economic Ideas, Princeton, Princeton University Press.

Kok W. (2003), Jobs, Jobs, Jobs: Creating More Employment in Europe, European Commission, Brussels.

Kok W. (2004), Facing the challenge: the Lisbon strategy for Growth and Employment, European Commission, Brussels.

Kröger S. (2009), "The Open Method of Coordination: Underconceptualisation, overdetermination, depoliticisation and beyond" in Kröger, Sandra (ed.): What we have learnt: Advances, pitfalls and remaining questions in OMC research, European Integration online Papers (EIoP), Special Issue 1, Vol. 13, Art. 5. http://eiop.or.at/eiop/texte/2009-005a.htm.

Muller P. (2000), «L'analyse cognitive des politiques publiques : vers une sociologie politique de l'action publique ", Revue Française de Science Politique, vol. $50, \mathrm{n}^{\circ} 2$, pp. 189-207.

North D. (1990), Institutions, Institutional Change and Economic Performance, Cambridge : Cambridge University Press.

Oates W. (1999), “An Essay on Fiscal Federalism”, Journal of Economic Literature, vol. 37, pp. 1120-1149.

Pisani Ferry J., Sapir A. (2006), Last exit to Lisbon, Bruegel Report, Brussels.

Rodriguez R., Warmerdam J., and Triomphe CE (dir, 2010), The Lisbon Strategy 2000 - 2010 An analysis and evaluation of the methods used and results achieved, European Parliament Policy reporthttp:// www.europarl.europa.eu/activities/committees/ studies $/$ searchPerform.do? page $=4 \&$ language $=\mathrm{EN}$

Sabel C., Zeitlin J. (2007), "Learning from Differences: the New Architecture of Experimentalist Governance in the European Union", European Governance Papers (EUROGOV), $\mathrm{n}^{\circ} \mathrm{C} 07-02$.

Trubek D., Trubek L. (2005), "Hard and Soft Law in the Construction of Social Europe: the Role of the Open Method of Coordination", European Law Journal, Vol. 11, n 3, may, pp. 343-364.

Wasmer E., Von Weizsäcker J. (2007), « Le fonds européen d'ajustement à la mondialisation : pour quoi faire ? ", Revue de l'OFCE, vol. 3, n 102, pp. 223-246.

Zeitlin J. (2005), "The Open Method of Coordination in Action, Theoretical Promise, Empirical Realities, Reform Strategies", in Pochet P., Zeitlin (dir), pp. 447-503.

Zeitlin J., Heidenreich M. (2009, eds), Changing European Employment and Welfare Regimes, London: Routledge. 
Résumé

\title{
La coordination européenne des politiques de l'emploi : un essouflement depuis 2005 ?
}

\author{
Christine Erhel
}

En 2005, la procédure de coordination des politiques de l'emploi au niveau de l'Union européenne a fait l'objet de réformes dans le cadre de la « relance " de la Stratégie de Lisbonne. On suppose ici que les effets de cette gouvernance européenne de l'emploi sont avant tout d'ordre institutionnel et cognitif. Dès lors, l'article analyse les transformations de la mise en œuvre de la Stratégie Européenne pour l'Emploi (SEE) dans la période qui suit ces réformes. L'analyse empirique se fonde sur une étude du cas français, et témoigne d'un affaiblissement de la sphère du travail et de l'emploi au profit de celle de l'économie. Des effets d'harmonisation cognitive via la SEE sont confirmés, mais demeurent graduels, sans accélération après 2005.

\section{Mots clés}

Politique de l'emploi, politique européenne, MOC - méthode ouverte de coordination -, France Journal of Economic Literature: : J 38, J 58 